\title{
Posibles estrategias de tratamiento para COVID-19
}

DOI: https://doi.org/10.5377/alerta.v3i2.9626

\section{José Eduardo Oliva Marín*}

Departamento de Gobernanza y Gestión del Conocimiento del Instituto Nacional de Salud, San Salvador, El Salvador.

${ }^{*}$ Correspondencia

$\square$ joseduardoliva67@gmail.com

(1) $0000-0002-6005-0558$

\section{Resumen}

No hay tratamiento antiviral específico para el COVID-19. Sin embargo, conocimientos adquiridos durante los brotes del SARS y el MERS, en conjunto con la información obtenida con COVID-19, han permitido detectar varios objetivos terapéuticos en el ciclo de replicación del virus, y en su patogénesis. Se incluye la evidencia actual con respecto a los principales tratamientos propuestos para COVID-19, reutilizados o experimentales, mediante una revisión de la literatura científica a la fecha. Debido a la falta de ensayos controlados aleatorios, se incluyeron: informes de casos, series de casos y artículos de revisión. Globalmente se están llevando a cabo múltiples estudios con el fin de identificar agentes que sean efectivos ante COVID-19, en los siguientes objetivos estratégicos: inhibición de la entrada/fusión del virus (anticuerpos neutralizantes, inhibidores de proteasa de serina transmembrana 2, cloroquina, hidroxicloroquina y umifenovir); interrupción de la replicación viral (remdesivir, favipiravir, lopinavir/ritonavir e ivermectina) y supresión de la respuesta inflamatoria excesiva (corticosteroides, tocilizumab, e inmunoglobulina). Aún no existe un tratamiento efectivo y seguro contra COVID-19; los fármacos descritos en esta revisión se administran como uso compasivo de drogas, o bien, como parte de un ensayo clínico. La terapia de soporte continúa siendo el pilar del manejo de COVID-19.

\section{Palabras clave}

Anticuerpos neutralizantes, hidroxicloroquina, lopinavir, ritonavir, interleucina-6.

\section{Abstract}

There is no specific antiviral treatment for COVID-19. However, knowledge acquired during the SARS and MERS outbreaks, together with the information obtained with COVID-19, have allowed the detection of various therapeutic targets in the virus replication cycle, and in its pathogenesis. The current evidence regarding the leading treatments proposed for COVID-19, reused or experimental, is included through a review of the scientific literature to date. Due to the lack of randomized controlled trials, the following were involved: case reports, case series and review articles. Globally, multiple studies are being carried out in order to identify agents that are effective against COVID-19, upon the following strategic objectives: inhibition of viral entry/fusion (neutralizing antibodies, transmembrane serine protease 2 inhibitors, chloroquine, hydroxychloroquine, and umifenovir); interruption of viral replication (remdesivir, favipiravir, lopinavir/ritonavir and ivermectin), and suppression of excessive inflammatory response (corticosteroids, tocilizumab, and immunoglobulin). There is still no effective and safe treatment against COVID-19; the medications described in this review are given as compassionate drug use, or as part of a clinical trial. Support therapy continues to be COVID-19 management cornerstone.

\section{Keywords}

Antibodies neutralizing, hydroxychloroquine, lopinavir-ritonavir, interleukin-6.

\section{ACCESO ABIERTO}

Possible treatment strategies for COVID-19

\section{Citación recomendada:}

Oliva Marín JE. Posibles estrategias de tratamiento para COVID-19.

Alerta 2020;3(2):87-100.

DOI: https://

doi.org/10.5377/alerta.

$\underline{\mathrm{v} 3 \mathrm{i} 2.9626}$

\section{Recibido:}

13 de abril 2020

\section{Aceptado:}

5 de mayo 2020

\section{Publicado:}

8 de mayo 2020

\section{Contribución de autoría:}

JEOM': elaboración del manuscrito y rendición de cuentas del mismo

\section{Conflicto de intereses:}

El autor declara no tener conflicto de intereses 


\section{Introducción}

Actualmente no hay un agente antiviral específico aprobado para COVID-19. El tratamiento de soporte, que incluye el mantenimiento de la vía aérea permeable, la oxigenoterapia, el manejo de los líquidos de mantenimiento, la nutrición y el uso de antibióticos de amplio espectro ante infecciones bacterianas secundarias, siguen siendo los pilares del manejo ${ }^{1}$.Se han detectado varios objetivos terapéuticos, tanto en el ciclo de replicación del SARS-CoV-2 como en su patogénesis. Con esto se determinará la utilidad de agentes antivirales ya existentes, o bien, se desarrollarán intervenciones nuevas, efectivas y seguras contra el virus.

Entre otros objetivos terapéuticos se encuentran: inhibición de la entrada/fusión del virus a la célula hospedero (anticuerpos neutralizantes, inhibidores de proteasa de serina transmembrana 2, cloroquina, hidroxicloroquina y umifenovir), interrupción de la replicación viral dentro de la célula hospedero (remdesivir, favipiravir, lopinavir/ ritonavir e ivermectina) y supresión de la respuesta inflamatoria excesiva (corticosteroides, tocilizumab e inmunoglobulina intravenosa). Actualmente se están realizando múltiples ensayos clínicos para evaluar la efectividad y seguridad de estas estrategias, consideradas como candidatas contra COVID-19² (Figura 1).

\section{Etapas de la enfermedad}

Las estrategias terapéuticas contra COVID-19 deben decidirse según la etapa de la infección. En la etapa I (fase leve) el virus se multiplica y coloniza el tracto respiratorio del hospedero, causando un cuadro gripal, con malestar general, fiebre y tos. El tratamiento actualmente es sintomático. El mejor momento para utilizar antivirales en COVID-19, si se aprueba alguno, es en la fase previa a la aceleración inmunológica, es decir, durante las etapas I y $\|^{3}$. Lo anterior, con el fin de reducir la duración de la sintomatología, prevenir la progresión a gravedad y disminuir el riesgo de contagio ${ }^{4}$.

La etapa II (fase moderada) se caracteriza por el desarrollo de neumonía, la cual puede ser leve, moderada o grave, ameritando ingreso para observación y tratamiento. El tratamiento consiste principalmente en medidas de soporte respiratorio y terapias antivirales disponibles (ya sea en la modalidad de uso compasivo de drogas o como parte de un ensayo (línico) ${ }^{4}$. En teoría, el efecto antiinflamatorio de los corticosteroides ayudaría a mejorar la evolución clínica de estos pacientes. Sin embargo, al momento no hay evidencia suficiente para recomendar el tratamiento con corticosteroides en pacientes con COVID-19,5.6 Su eficacia y seguridad se determinarán en los ensayos controlados aleatorizados en proceso.

La etapa III (fase grave) se manifiesta como un síndrome de hiperinflamación sistémica extrapulmonar. En esta etapa, el shock, la vasoplejía, la insuficiencia respiratoria, la afectación sistémica de órganos e incluso el colapso cardiopulmonar, son evidentes. Para la etapa III, el uso de inmunomoduladores es la propuesta, con el fin de disminuir la aceleración inmunológica que la caracteriza ${ }^{4}$. Se ha sugerido el uso de corticosteroides e inhibidores de las interleucinas 1 y 6, como anakinra y tocilizumab, respectivamente ${ }^{7}$. El uso de inmunoglobulina intravenosa (IGIV) también puede desempeñar un papel inmunomodulador durante esta fase ${ }^{3,4,8}$.

\section{Inhibición de la entrada/ fusión}

\section{Desarrollo de anticuerpos neutralizantes contra SARS-CoV-2}

Se trata del desarrollo de un anticuerpo neutralizante dirigido a la proteína espiga $\mathrm{S}$ del SARS-CoV-2, proporcionando inmunidad pasiva a la enfermedad. El desafío es que cualquier anticuerpo candidato necesita ser rigurosamente probado en cultivos celulares y modelos animales para confirmar su eficacia y seguridad, antes de ser utilizado en humanos. La información conocida del virus SARS-CoV-2 es útil al momento de decidir qué epítopo de anticuerpos neutralizantes (la unión de la proteína espiga S es el primer objetivo clave) se debe producir?. Este es un proceso lento y desafiante, que puede no rendir resultados significativos durante varios meses.

\section{Anticuerpos neutralizantes presentes en plasma de pacientes convalecientes}

El uso de plasma de pacientes convalecientes con anticuerpos formados durante la evolución de la enfermedad no es nuevo. Esto fue utilizado durante el brote de SARS en 2002; el brote de influenza aviar A en 1997(H5N1); la pandemia de influenza A en 2009 (H1N1) y brotes de fiebres hemorrágicas como el Ébola y otras infecciones virales ${ }^{10}$.

Shen et al., en marzo de $2020^{11}$, publicó una serie de casos no controlados de cinco enfermos críticos con COVID-19, quienes desarrollaron síndrome de dificultad respiratoria aguda (SDRA). Se les administró plasma 


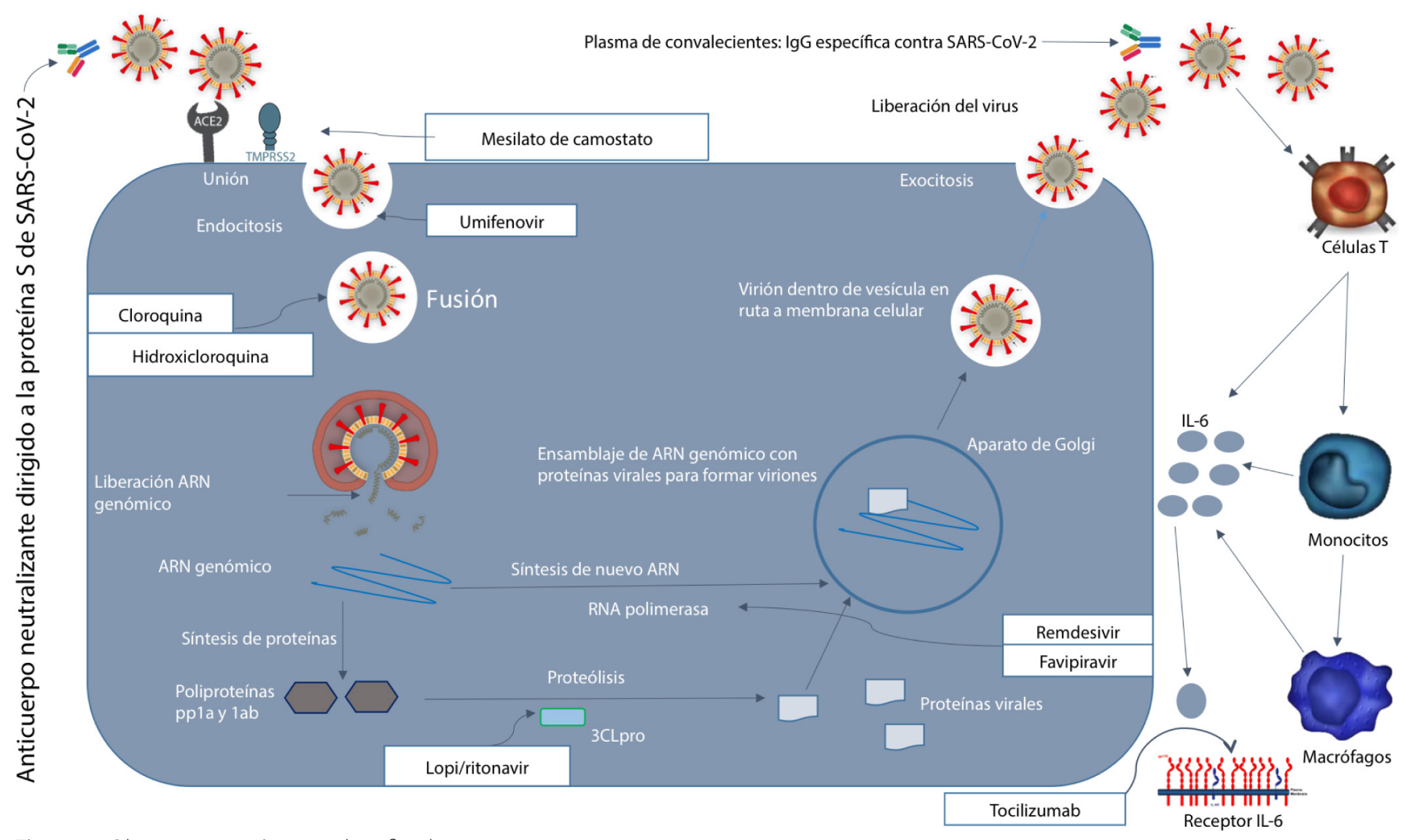

Figura 1. Objetivos terapéuticos identificados

obtenido de pacientes convalecientes de COVID-19, conteniendo anticuerpos neutralizantes IgG específicos contra SARSCoV-2, con lo que se obtuvo mejoría de su estado clínico. Tanto el tamaño limitado de la muestra como el diseño del estudio, impiden que se dé una recomendación definitiva sobre el uso de esta estrategia ante casos graves de COVID-19. Estas recomendaciones requieren una evaluación rigurosa y exhaustiva mediante ensayos controlados aleatorizados.

\section{Agentes que bloquean al receptor ECA2}

Las tres estrategias propuestas bloquean la interacción de la proteína espiga S/receptor ECA2 celular y anulan la infección (Figura 2). La primera consiste en administrar al paciente grandes cantidades de dominio de unión al receptor (RBD, por sus siglas en inglés) de la proteína espiga S del SARS-CoV o del SARS-CoV-2. Este RBD soluble se une a los receptores ECA2 celulares, saturando así los sitios disponibles para el acoplamiento del virus. Alternativamente, un anticuerpo o un fragmento variable de anticuerpo de cadena simple (scFv, por sus siglas en inglés), podrían administrarse a los pacientes para que se unan a los receptores ECA2, logrando el mismo efecto. Una tercera estrategia apunta directamente a los viriones del SARS-CoV-2, mediante el uso de una molécula similar a un anticuerpo que se une al virus, no per- mitiendo el acople de este al receptor ECA2. Para esta estrategia, se utiliza un complejo compuesto por una versión soluble del receptor ECA2 unido a un scFv (ECA2-Fc). Este complejo se une a la proteína espiga $S$ del SARS-COV-2 y no permite la unión de esta con el receptor ECA2 ${ }^{9}$. Cualquiera que sea la estrategia a utilizar, lo recomendable es emplearla tempranamente durante la infección, o como profilaxis, para bloquear la infección viral inicial. Se necesita comprobar la eficacia y seguridad de estas estrategias con ensayos controlados aleatorizados.

\section{Inhibidores de proteasa de serina transmembrana 2}

La entrada a las células hospedero no solo depende de la unión de la proteína espiga $S$ con los receptores ECA2, sino también de la actividad de la enzima proteasa de serina transmembrana 2 (TMPRSS2, por sus siglas en inglés). TMPRSS2 escinde proteolíticamente y activa la proteína espiga S, lo que facilita la entrada y fusión del virus con la membrana celular. El inhibidor de proteasa de serina, mesilato de camostato, ha mostrado efectividad in vitro contra SARS-CoV, NL63 y, actualmente, contra SARS-CoV-2, reduciendo significativamente la infección en los cultivos de células pulmonares ${ }^{12}$. Por lo anterior, podría considerarse para estudios clínicos en la búsqueda de un tratamiento para COVID-19. 


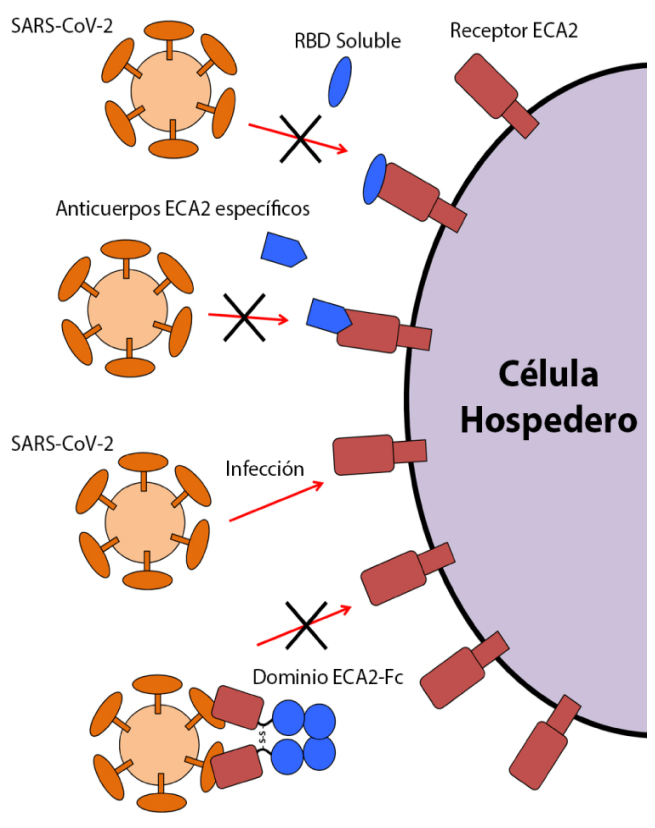

Figura 2. Agentes que bloquean al receptor ECA2.

Tomado de: Kruse RL. Therapeutic strategies in an outbreak scenario to treat the novel coronavirus originating in Wuhan, China. F1000Research. 2020; 9:72. DOl: https://doi.org/10.12688/ f1000research.22211.2. Se efectuaron modificaciones. https://creativecommons.org/licenses/by/4.0/

\section{Cloroquina e hidroxicloroquina}

La cloroquina y la hidroxicloroquina, antipalúdicos y moduladores autoinmunes, han sido consideradas recientemente como medicamentos que cuentan con un amplio potencial antiviral. En estudios previos con SARS-CoV, se determinó que la cloroquina bloquea la infección viral al aumentar el pH endosómico requerido para la fusión virus/ membrana celular ${ }^{13}$. Se ha planteado, además, la hipótesis que la cloroquina podría bloquear la producción de citocinas proinflamatorias como la interleucina-6, interrumpiendo así la aceleración inmunológica que eventualmente conduce al SDRA ${ }^{14}$. Los ensayos controlados aleatorizados en curso en todo el mundo, con cloroquina e hidroxicloroquina, constatarán si ambas son eficaces y seguras como tratamiento para COVID-19.

El 28 de marzo de 2020, la Food and Drug Administration (FDA) emitió una autorización de uso de emergencia para permitir que la hidroxicloroquina y la cloroquina se utilizaran en pacientes hospitalizados con COVID-19, cuando un ensayo clínico no estuviese disponible o no fuese factible ${ }^{15}$. Desde su autorización para uso de emergencia en pacientes con COVID-19, la FDA ha recibido informes de eventos adversos cardíacos en pacientes con COVID-19 que recibieron hidroxicloroquina o cloroquina, ya sea como monoterapia o en combinación con azitromicina u otros medicamentos. Estos eventos adversos incluyen la prolongación del intervalo QT, la taquicardia ventricular, la fibrilación ventricular e incluso la muerte. La FDA continúa investigando estos riesgos de seguridad en pacientes con COVID-1916.

El 24 de abril de 2020 la FDA publicó una alerta contra el uso de la hidroxicloroquina o la cloroquina en pacientes COVID-19 no hospitalizados, y contra el uso de ambos fármacos como profilaxis de esta enfermedad. Recalcan en esta alerta que autorizan el uso temporal de ambos fármacos solo en pacientes hospitalizados, o bien, inscritos en un ensayo clínico, para una detección temprana de complicaciones a través de un monitoreo apropiado ${ }^{16}$. La monitorización debe incluir la evaluación de antecedentes cardiovasculares, ECG basal, electrolitos, función renal y pruebas hepáticas.

El 15 de junio de 2020 la FDA prohibió el uso de la cloroquina y la hidroxicloroquina como profilaxis o tratamiento de COVID-19.

\section{Umifenovir}

Umifenovir es un potente antiviral de amplio espectro de fabricación rusa, inhibe el paso inicial de entrada/fusión de algunos virus a las células del hospedero ${ }^{17}$. Estudios in vitro con SARS-CoV sugieren un efecto directo en la replicación viral temprana al insertarlo en los cultivos celulares. Actualmente, no existen datos clínicos que avalen su eficacia y seguridad como terapéutico contra COVID-19'18.

\section{Interrupción de la replicación}

\section{Remdesivir}

Remdesivir es un medicamento experimental, desarrollado en 2017 por Gilead Sciences como tratamiento para la enfermedad por el virus del Ébola y las infecciones por el virus de Marburg ${ }^{19}$. Es un profármaco de un análogo de nucleótido que se metaboliza intracelularmente a un análogo de trifosfato de adenosina, que inhibe las ARN polimerasas virales. Específicamente, se metaboliza en su forma activa, GS-441524, que bloquea una enzima muy particular que se requiere para la replicación viral, la ARN polimerasa dependiente de ARN, causando una disminución en la producción de ARN viral20.

Recientemente Wang et al., utilizando cultivos de células Vero E6, demostró que 
remdesivir inhibió in vitro la replicación del SARS-CoV-22. En macacos Rhesus infectados con SARS-CoV-2, el tratamiento con un régimen de remdesivir IV de 6 días, ini ciado 12 horas después de la inoculación del virus, se asoció con algunos beneficios (puntuaciones más bajas de gravedad de la enfermedad, menos infiltrados pulmonares y títulos más bajos del virus en muestras de lavado broncoalveolar), en comparación con el grupo control. Sin embargo, remdesivir no redujo las cargas virales en la nariz, la garganta e hisopos rectales, al comparar con el grupo control ${ }^{22}$. A principios de abril 2020, Grein et al. ${ }^{23}$ reportó una cohorte de 53 pacientes hospitalizados por COVID-19 grave, tratados con remdesivir por razones compasivas, observando mejoría clínica en 36 de ellos (68\%). La corroboración de esta eficacia requerirá de ensayos controlados aleatorizados.

El 1 de mayo de 2020, la FDA emitió una autorización de uso de emergencia para remdesivir para el tratamiento de adultos y niños hospitalizados por COVID-19 grave. Si bien la información sobre la eficacia y la seguridad del remdesivir en el tratamiento de pacientes ingresados con COVID-19 es escasa, datos preliminares del ensayo controlado aleatorizado Adaptive COVID-19 Treatment Trial (ACTT), del National Institute of Allergy and Infectious Diseases (NIAID) de Estados Unidos, orientan a que el fármaco acorta el tiempo de recuperación en algunos pacientes. Es con base en estos datos preliminares, que la FDA emitió la autorización ya descrita ${ }^{24}$.

\section{Favipiravir}

Fue aprobado para el tratamiento de la influenza el 15 de febrero de 2020 en China. Es un nuevo tipo de inhibidor de la ARN polimerasa dependiente de ARN. El favipiravir se convierte en su forma fosforibosilada activa (favipiravir-RTP) dentro de las células e inhibe la actividad de la ARN polimerasa viral ${ }^{25}$. Por lo anterior, el favipiravir puede tener una posible acción antiviral sobre el SARS-CoV-2, siendo este un virus ARN ${ }^{26}$. Sin embargo, es un mutágeno y tiene potencial, tanto para la teratogenicidad como para la embriotoxicidad en humanos. El 1 de junio de 2020 favipiravir recibió un certificado de registro temporal del Ministerio de Salud de la Federación Rusa, como droga terapéutica contra COVID-19.

\section{Lopinavir/ritonavir}

Inhibidores de la proteasa del virus de la inmunodeficiencia humana $(\mathrm{VIH})$, inhiben la proteinasa principal de SARS-CoV y MERSCoV, la proteasa similar a 3-quimotripsina (3CLpro, por sus siglas en inglés). La 3CLpro es responsable de la proteólisis mediante la cual las poliproteínas pp1a y 1ab se subdividen en proteínas más pequeñas y funcionales, las cuales posteriormente se utilizan en el ensamblaje de los nuevos viriones ${ }^{27}$. La Organización Mundial de la Salud (OMS) ha manifestado que esta combinación podría proporcionar algún beneficio clínico ante COVID-1928.

\section{Ivermectina}

El proceso de entrada de algunos virus ARN al núcleo de la célula hospedero, es facilitado por las proteínas IMP (importinas). La ivermectina ha sido identificada como un inhibidor potencial de la importación nuclear de proteínas virales, mediada por una de estas importinas, la proteína IMPa/ $\beta 1$. Al no poder acceder al genoma viral al núcleo de la célula hospedero, la replicación viral no se inicia. La confirmación de este mecanismo de acción ante el SARS-CoV-2, es un enfoque importante a tomar en cuenta para futuros estudios ${ }^{29}$.

En un estudio in vitro reciente, llevado a cabo por Caly et al. en Australia29, se observó una reducción del $93 \%$ del ARN viral presente en los cultivos celulares en 24 horas, al ser tratados con ivermectina. A las 48 horas, se observó una reducción del $99.8 \%$. El tratamiento con ivermectina resultó en la pérdida efectiva de casi todo el material viral en 48 horas. Parece que la actividad inhibidora del transporte nuclear de la ivermectina, puede ser efectiva ante el SARS-CoV-2. En conjunto, los hallazgos de este estudio con su perfil de seguridad conocido, orientan a que la ivermectina merece ser considerada como un posible antiviral contra SARS-CoV-2. Su uso para tal fin, dependerá de la realización de ensayos controlados aleatorizados.

El 19 de junio de 2020 la Organización Panamerica na de la Salud emitió una advertencia contra el uso de la ivermectina como terapéutico contra COVID-19.

\section{Supresión de la respuesta inflamatoria excesiva}

\section{Corticosteroides}

Ante cualquier proceso infeccioso es esencial que un hospedero presente una respuesta coordinada de citocinas; sin embargo, una respuesta exagerada conduce a una condición hiperinflamatoria, tal como se ha detectado en pacientes graves infectados con SARS-CoV-2 ${ }^{30}$. En teoría, el tratamiento 
con corticosteroides durante la fase de SDRA podría inhibir efectivamente la tormenta de citocinas, y ganar tiempo para controlar la infección y prevenir los daños secundarios, como la falla multiorgánica.

Zhou et al. ${ }^{31}$, en un estudio publicado en febrero de 2020, que incluyó a 15 pacientes con neumonía grave por COVID-19, concluyó que los corticosteroides no ejercieron ningún beneficio en la supervivencia de estos pacientes, quienes, a la vez, estaban complicados con SDRA, shock y/o lesión de múltiples órganos. Se necesitan con urgencia datos de ensayos clínicos aleatorios y controlados, para definir claramente el papel de los corticosteroides en el tratamiento de COVID-1918.

La dexametasona se ha investigado desde marzo de 2020, en pacientes hospitalizados reclutados en el ensayo controlado aleatorizado Randomized Evaluation of Covid-19 Therapy (RECOVERY), del Reino Unido, y se descubrió que tiene beneficios para los pacientes críticos. Según los hallazgos preliminares compartidos con la OMS para los pacientes ventilados, se demostró que el tratamiento reduce la mortalidad en aproximadamente un tercio, y para los pacientes que solo requieren oxígeno, la mortalidad se redujo en aproximadamente un quinto.

\section{Tocilizumab}

Tocilizumab es un anticuerpo monoclonal humanizado, recombinante, que se une específicamente a los receptores de la IL-6, disminuyendo así su acción. La IL-6 ha sido identificada como la molécula clave en la tormenta de citocinas que caracteriza a las formas graves de COVID-19. Por lo anterior, un antagonista de los receptores de la IL-6 como el tocilizumab, debe tomarse en cuenta como posible terapéutico ante los casos graves de COVID-1932.

\section{Inmunoglobulina intravenosa (IGIV)}

La IGIV es un producto sanguíneo que contiene inmunoglobulina policlonal $\mathrm{G}$ aislada de donantes sanos. Ha sido utilizada por más de 30 años como terapia inmunomoduladora para enfermedades autoinmunes o inflamatorias y para la profilaxis y el tratamiento de infecciones graves, especialmente en pacientes inmunocomprometidos. Es un producto diferente al plasma de pacientes convalecientes de COVID-19, el cual contiene lgG específica para SARS-CoV-2. La IGIV puede modular la respuesta inmune a través de múltiples mecanismos, incluidos el bloqueo de un amplio conjunto de citocinas proinflamatorias, el bloqueo a los recep- tores gamma de la porción Fc de las IgG's y a las moléculas de adhesión leucocitaria. En COVID-19 los primeros días de deterioro presentan un punto crítico en la evolución clínica del paciente. Es una ventana de tiempo durante la cual una supresión potente de la cascada inflamatoria, podría salvar a los pacientes del deterioro clínico fatal mediado por el sistema inmune ${ }^{3}$.

Considerando su eficacia para mejorar la inmunidad pasiva y modular la inflamación inmune, así como su perfil general de seguridad, la IGIV podría considerarse como una opción prometedora en la etapa inicial del deterioro clínico de los pacientes con COVID-19, inhibiendo la cascada inflamatoria que caracteriza a los casos graves.

\section{Miscelaneos}

\section{Otros antivirales}

Existen muchos otros medicamentos en investigación para determinar si son eficaces o no contra el SARS-CoV-2, discutidos ampliamente en la literatura, como la ribavirina, el interferón, el oseltamivir, la nitazoxanida, la teicoplanina, la emetina y la homoharringtonina. En el caso de los primeros tres, se ha evidenciado que los riesgos superan los beneficios ${ }^{18}$, y en el caso de la nitazoxanida ${ }^{21}$, la teicoplanina ${ }^{33}$, la emetina ${ }^{34}$ y la homoharringtonina ${ }^{34}$, a la fecha han demostrado su efectividad contra el SARS-CoV-2 in vitro, pero hacen falta los ensayos controlados aleatorizados correspondientes, que comprueben su eficacia y seguridad contra COVID-19.

\section{Antiinflamatorios no esteroideos (AINE)}

La OMS refiere que para los pacientes que utilizan crónicamente algún AINE, en la actualidad no hay evidencia de eventos adversos graves, necesidad de atención médica de emergencia, disminución de la supervivencia a largo plazo o menor calidad de vida, si desarrollan COVID-1935. Por lo que, mientras se generan evidencias adicionales, la OMS considera adecuada la aproximación sugerida por el National Health Service del Reino Unido (UK-NHS), quien no aconseja a los pacientes suspender tratamientos crónicos de AINE al desarrollar COVID-19. En el caso de personas que no utilizan AINE de forma crónica, el UK-NHS sugiere el uso de paracetamol o de ibuprofeno para tratar los síntomas de COVID-19, priorizando el uso del paracetamol, tomando en cuenta que tiene menos efectos secundarios que los AINE y es la opción más segura para la mayoría de las personas ${ }^{36}$. 
La European Medicines Agency (EMA) recomienda que al comenzar el tratamiento para la fiebre o el dolor en COVID-19, los pacientes y los profesionales de la salud deben considerar todas las opciones de tratamientos disponibles, incluidos paracetamol (acetaminofén) y AINE. Cada medicamento tiene sus propios beneficios y riesgos que se reflejan en la información del producto, y que deben considerarse junto con las pautas nacionales de tratamiento de cada país, en donde la mayoría recomienda el paracetamol como primera opción de tratamiento para la fiebre o el dolor ${ }^{37}$.

Al momento, la FDA no tiene conocimiento de evidencia científica que relacione el uso de algún AINE con el empeoramiento de los síntomas de COVID-19. Sin embargo, advierte que la actividad farmacológica de los AINE, que reduce la inflamación, y posiblemente la fiebre, puede enmascarar los signos físicos que ayudan al diagnóstico oportuno de algunas enfermedades infecciosas $^{38}$.

\section{Anticoagulación}

La COVID-19 puede predisponer al desarrollo de una enfermedad tromboembólica venosa y arterial debido a inflamación excesiva, hipoxia, inmovilización y coagulación intravascular diseminada (CID) ${ }^{39-41}$. El conocimiento preciso de la incidencia de complicaciones trombóticas en los pacientes con COVID-19, es importante para la toma de decisiones con respecto a la intensidad de la tromboprofilaxis, especialmente en pacientes ingresados en Unidades de Cuidados Intensivos. La aplicación activa de anticoagulantes (como la heparina) en pacientes con COVID-19 grave ha sido recomendada ampliamente ${ }^{42}$. El tratamiento con heparina parece estar asociado a un mejor pronós- tico en los pacientes graves con COVID-19 que presentan coagulopatía; sin embargo, su eficacia aún está por validarse mediante ensayos controlados aleatorizados ${ }^{43}$.

\section{Uso compasivo de drogas}

Muchos países han decidido incluir estos medicamentos en investigación en sus protocolos de atención, tomando en cuenta que en el contexto de un brote caracterizado por una alta mortalidad, puede ser éticamente correcto ofrecer individualmente a los pacientes, fármacos experimentales de emergencia, fuera del contexto o ámbito de una investigación, denominándose dicha conducta como uso compasivo de drogas ${ }^{44}$.

Para llevar a cabo esta conducta éticamente, se deben cumplir los siguientes siete requisitos de la OMS: 1) no existe un tratamiento efectivo aprobado; 2) no es posible iniciar estudios clínicos inmediatamente; 3) existencia de datos que proporcionen soporte preliminar de la eficacia y la seguridad del fármaco a utilizar, al menos de estudios in vitro o en modelos animales, y que su uso fuera del ámbito de la investigación, ha sido sugerido por un comité científico debidamente calificado, sobre la base de un análisis riesgo/beneficio favorable; 4) que el fármaco a utilizar cuente con el aval de las autoridades relevantes del país, así como de un comité de ética debidamente calificado; 5) que el país cuente con recursos adecuados para asegurar que los riesgos que puedan surgir por el uso del fármaco, sean disminuidos; 6) se obtiene el consentimiento informado firmado por el paciente o su representante; y 7) el uso de emergencia del fármaco es monitoreado, los resultados documentados y eventualmente compartidos de manera oportuna, con la comunidad médica y científica ${ }^{44}$. 
Tabla1. Principales medicamentos en investigación para COVID-19

\begin{tabular}{ll}
\hline Droga & Mecanismo de acción \\
\hline Cloroquina: & Bloquea la infección \\
Antimalárico & viral al aumentar el pH \\
e inmunomodulador & endosómico requerido \\
& para la fusión virus/ \\
& membrana celular ${ }^{13}$. \\
& \\
& Bloquea la producción \\
& de citocinas \\
& proinflamatorias como \\
& la interleucina-6, \\
& interrumpiendo \\
& así la aceleración \\
& inmunológica que \\
& eventualmente \\
& conduce al SDRA ${ }^{14}$.
\end{tabular}

Fundamento

Se ha reportado actividad in vitro contra SARS-CoV-2 en células Vero E6 infectadas; así como alguna evidencia de que puede bloquear la entrada a las células Vero E6, al ser estas expuestas al SARS-COV-2 ${ }^{21}$

La eficacia y seguridad de la cloroquina para el tratamiento o la prevención de COVID-19 no se han establecido ${ }^{15,16}$.

Las guías de tratamiento para COVID-19 de los NIH ${ }^{53}$, establecen que los datos clínicos son insuficientes al momento para recomendar, a favor o en contra, sobre el uso de la cloroquina como tratamiento para COVID-19.

Las guías de tratamiento y manejo para pacientes con COVID-19 IDSA ${ }^{54}$, recomiendan que se use cloroquina para el tratamiento de COVID-19, solo en el contexto de un ensayo clínico.

Las guías de tratamiento para COVID-19 de los $\mathrm{NIH}^{53}$, no recomiendan el uso de ningún agente, incluida la cloroquina, para la profilaxis previa a la exposición (PrEP) o la profilaxis posterior a la exposición (PEP), para la prevención de la infección por SARS-CoV-2 fuera del contexto de un ensayo clínico.

Debido a que la cloroquina está asociada con la prolongación del intervalo QT, se recomienda precaución en pacientes con riesgo de prolongación del intervalo QT o que estén recibiendo otros medicamentos asociados con arritmias; se recomiendan pruebas de diagnóstico y monitoreo para minimizar el riesgo de efectos adversos, incluidos los efectos cardíacos inducidos por fármacos ${ }^{15,16}$

\begin{tabular}{ll}
\hline Hidroxicloroquina: & Incrementa el pH \\
Fármaco & intracelular e inhibe la \\
antirreumático & actividad lisosómica \\
& en las células \\
& presentadoras de \\
& antígenos, incluidas \\
& las células dendríticas \\
& plasmacitoides y las \\
& células B, lo que evita \\
& el procesamiento \\
& de antígenos y la \\
& presentación de \\
& autoantígenos a \\
& las células T, esta \\
& última mediada \\
& por el complejo de \\
& histocompatibilidad \\
& clase Il ${ }^{45}$. \\
\end{tabular}

\section{Se ha reportado actividad in vitro contra SARS-CoV-2 en células Vero E6 infectadas; puede ser más potente que la cloroquina in vitro, pero algunos datos son contradictorios y se necesitan estudios adicionales ${ }^{46,47}$.}

La eficacia y la seguridad de la hidroxicloroquina, en el tratamiento o prevención de COVID-19, no se han establecido ${ }^{15-16}$. Las guías de tratamiento para COVID-19 de los $\mathrm{NIH}^{53}$, establecen que los datos clínicos son insuficientes para recomendar, a favor o en contra, sobre el uso de la hidroxicloroquina como tratamiento para COVID-19.

Las guías de tratamiento y manejo para pacientes con COVID-19 IDSA $^{54}$, recomiendan que la hidroxicloroquina se use para el tratamiento de COVID-19 solo en el contexto de un ensayo clínico.

Las guías de tratamiento para COVID-19 de los NIH ${ }^{53}$, recomiendan no usar un régimen combinado de hidroxicloroquina y azitromicina para el tratamiento de COVID-19, excepto en el contexto de un ensayo clínico.

Las guías de tratamiento y manejo para pacientes con COVID-19 IDSA $^{54}$, recomiendan que, de utilizarse un régimen combinado de hidroxicloroquina y azitromicina para el tratamiento de COVID-19, sea solo en el contexto de un ensayo clínico.

Las guías de tratamiento para COVID-19 de los $\mathrm{NIH}^{53}$, no recomiendan el uso de ningún agente, incluido la hidroxicloroquina, para la PrEP o la PEP, para la prevención de la infección por SARS-CoV-2 fuera del contexto de un ensayo clínico.

Aplican las mismas precauciones sobre la prolongación del intervalo QT descritas en el apartado de cloroquina. 


\begin{tabular}{|c|c|c|c|}
\hline Droga & Mecanismo de acción & Fundamento & Comentarios \\
\hline $\begin{array}{l}\text { Azitromicina: } \\
\text { Macrólido }\end{array}$ & $\begin{array}{l}\text { Inhibe la síntesis de } \\
\text { proteínas dependiente } \\
\text { de ARN } \\
\text { Multiples efectos } \\
\text { inmunomoduladores }{ }^{48} \text {. }\end{array}$ & $\begin{array}{l}\text { A la fecha, no hay datos } \\
\text { sobre actividad in vitro } \\
\text { contra coronavirus, } \\
\text { incluido el SARS-CoV-2. } \\
\text { Antibacteriano con } \\
\text { actividad in vitro contra } \\
\text { algunos virus (p. ej., } \\
\text { influenza A H1N1, zika) } \\
49,50\end{array}$ & $\begin{array}{l}\text { Las guías de tratamiento para COVID-19 de los } \mathrm{NIH}^{53} \text {, } \\
\text { recomiendan no usar un régimen combinado de } \\
\text { hidroxicloroquina y azitromicina para el tratamiento de COVID-19, } \\
\text { excepto en el contexto de un ensayo clínico. } \\
\text { Las guías de tratamiento y manejo para pacientes con } \\
\text { CoVID-19 IDSA (2020) }{ }^{54} \text {, recomiendan que, de utilizarse un } \\
\text { régimen combinado de hidroxicloroquina y azitromicina para el } \\
\text { tratamiento de COVID-19, sea solo en el contexto de un ensayo } \\
\text { clínico. } \\
\text { Aplican las mismas precauciones sobre la prolongación del } \\
\text { intervalo QT descritas en el apartado de cloroquina. }\end{array}$ \\
\hline $\begin{array}{l}\text { Remdesivir: } \\
\text { Antiviral } \\
\text { análogo } \\
\text { nucléosido }\end{array}$ & $\begin{array}{l}\text { Inhibidor de la ARN } \\
\text { polimerasa viral }{ }^{20} \text {. }\end{array}$ & $\begin{array}{l}\text { Evidencia de actividad } \\
\text { in vitro contra SARS- } \\
\text { CoV-2 en células } \\
\text { Vero E6 } 6^{21} \text {; así como } \\
\text { en macacos Rhesus } \\
\text { infectados². }\end{array}$ & 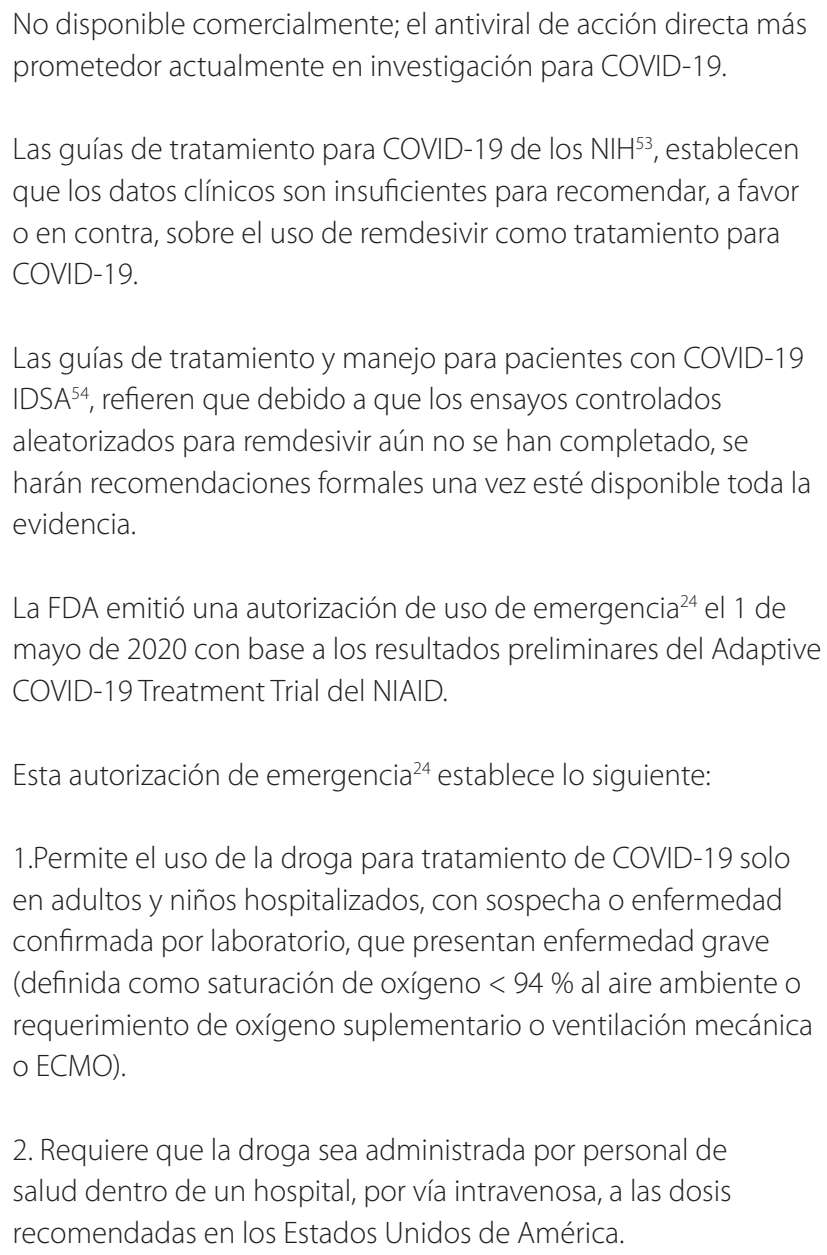 \\
\hline $\begin{array}{l}\text { Lopinavir/ritonavir: } \\
\text { Antirretrovirales }\end{array}$ & $\begin{array}{l}\text { Inhibidores de } \\
\text { proteasa del virus de } \\
\text { inmunodeficiencia } \\
\text { humana }{ }^{18} \text {. }\end{array}$ & $\begin{array}{l}\text { Lopinavir (LPV): se ha } \\
\text { reportado actividad in } \\
\text { vitro contra SARS- } \\
\text { CoV-2 en células Vero } \\
\text { E6 }{ }^{34} \text {. }\end{array}$ & $\begin{array}{l}\text { Las guías de tratamiento para COVID-19 de los } \mathrm{NIH}^{53} \text {, } \\
\text { recomiendan no usar LPV/RTV u otros inhibidores de la proteasa } \\
\text { del VIH para el tratamiento de COVID-19, excepto en el contexto } \\
\text { de un ensayo clínico. } \\
\text { Las guías de tratamiento y manejo para pacientes con COVID-19 } \\
\text { IDSA }{ }^{54} \text {, recomiendan que, de utilizar LPV/RTV para el tratamiento } \\
\text { de COVID-19, se haga solo en el contexto de un ensayo clínico. }\end{array}$ \\
\hline
\end{tabular}




\begin{tabular}{llll}
\hline Droga & Mecanismo de acción & Fundamento & Comentarios \\
\hline Metilprednisolona: & Supresión de & Potentes propiedades & Hallazgos de estudios observacionales sugieren que para \\
Corticosteroide & exuberante de la & antiinflamatorias y & pacientes con COVID-19 y neumonía que progresa a SDRA, el \\
& inflamación sistémica & antifibróticas. El uso & tratamiento con metilprednisolona puede ser beneficioso. Sin \\
& disfuncional ${ }^{30-31}$. & de corticosteroides & embargo, los resultados deben ser interpretados con precaución \\
& puede prevenir una & debido al sesgo potencial (fármaco utilizado en pacientes más \\
& respuesta extensa & enfermos) y al tamaño pequeño de las muestras. Es necesaria la \\
& de citocinas y puede & confirmación mediante estudios controlados aleatorizados ${ }^{5,6}$.
\end{tabular}

de la inflamación

pulmonar y sistémica,

en pacientes que

desarrollan neu-

monía 5 .

Para pacientes críticos, las guías de tratamiento para COVID-19 de los $\mathrm{NIH}^{53}$, establecen:

1. No recomiendan el uso rutinario de corticosteroides sistémicos para el tratamiento de pacientes COVID-19 en ventilación mecánica, si estos no han desarrollado síndrome de dificultad respiratoria aguda (SDRA).

2. Para pacientes en ventilación mecánica que han desarrollado SDRA, no hay pruebas suficientes para recomendar, a favor o en contra, sobre el uso de corticosteroides sistémicos.

3. Para los adultos con COVID-19 y shock refractario, recomiendan el uso de dosis bajas de corticosteroides.

Las guías de tratamiento y manejo para pacientes con COVID-19 IDSA ${ }^{54}$, no recomiendan el uso de corticosteroides en pacientes ingresados por neumonía por COVID-19.

Las guías de tratamiento y manejo para pacientes con COVID-19 IDSA ${ }^{54}$, recomiendan el uso de corticosteroides para aquellos pacientes ingresados por COVID-19 que han desarrollado SDRA, si se efectúa en el contexto de un ensayo clínico.

\begin{tabular}{lll}
\hline Tocilizumab: & Inhibe la IL-6y la & Puede interrumpir, \\
Anticuerpo & tormenta inflamatoria & $\begin{array}{l}\text { potencialmente, el } \\
\text { monoclonal }\end{array}$ \\
de citocinas ${ }^{32}$. & $\begin{array}{l}\text { síndrome de liberación } \\
\text { de citocinas (SRC), } \\
\text { recombinante, }\end{array}$ & en pacientes con \\
específico para & & COVID-19 gravemente \\
el receptor de la & & enfermos $^{7}$.
\end{tabular}

interleucina-6 (IL-6)

\section{Plasma de paciente} convaleciente de COVID-19

\section{Inmunidad pasiva al} contener lgG específica contra SARS-CoV-2, producida por el donador durante su enfermedad ${ }^{10,11}$

\section{En teoría, el plasma} obtenido de pacientes que se han recuperado de COVID-19, contiene anticuerpos lgG contra SARS-CoV-2, pudiendo proporcionar inmunidad pasiva a corto plazo que podría prevenir la infección; o bien, ser beneficioso como tratamiento en pacientes que están adoleciendo la enfermedad ${ }^{10,11}$.
Las guías de tratamiento para COVID-19 de los $\mathrm{NIH}^{53}$, afirman que no hay datos clínicos suficientes para recomendar, a favor o en contra, sobre el uso de tocilizumab como tratamiento para COVID-19.

Las guías de tratamiento y manejo para pacientes con COVID-19 IDSA ${ }^{54}$, recomiendan, para los pacientes ingresados por COVID-19, el uso de tocilizumab solo en el contexto de un ensayo clínico.

La eficacia y seguridad del plasma convaleciente COVID-19, para el tratamiento de COVID-19, no se han establecido ${ }^{55}$.

Las guías de tratamiento para COVID-19 de los $\mathrm{NIH}^{53}$, indican que no hay datos clínicos suficientes para recomendar, a favor o en contra, sobre el uso de plasma convaleciente COVID-19 o la inmunoglobulina humana, como tratamientos para COVID-19. Las guías de tratamiento y manejo para pacientes con COVID-19 IDSA $^{54}$, recomiendan, para los pacientes ingresados por COVID-19, el uso de plasma convaleciente COVID-19 solo en el contexto de un ensayo clínico. 


\begin{tabular}{|c|c|c|c|}
\hline Droga & Mecanismo de acción & Fundamento & Comentarios \\
\hline $\begin{array}{l}\text { Anticoagulantes: } \\
\text { (heparina de bajo } \\
\text { peso molecular } \\
\text { [HBPM], heparina no } \\
\text { fraccionada [UFH]) }\end{array}$ & $\begin{array}{l}\text { Inhibición de la vía del } \\
\text { factor tisular }{ }^{43} \text {. }\end{array}$ & $\begin{array}{l}\text { Un hallazgo constante } \\
\text { en pacientes con } \\
\text { COVID-19 grave } \\
\text { es un estado de } \\
\text { hipercoagulabilidad, } \\
\text { que puede contribuir } \\
\text { a un mayor riesgo de } \\
\text { complicaciones }{ }^{51,52} \text {. } \\
\text { Las anomalías de } \\
\text { la coagulación } \\
\text { observadas en estos } \\
\text { pacientes incluyen } \\
\text { la coagulación } \\
\text { intravascular } \\
\text { diseminada } \\
\text { protrombótica (CID), } \\
\text { una alta incidencia de } \\
\text { tromboembolismo } \\
\text { venoso, niveles } \\
\text { elevados de dímero } \\
\text { D, niveles altos } \\
\text { de fibrinógeno } \\
\text { y trombosis } \\
\text { microvascular y/o } \\
\text { macrovascular }\end{array}$ & $\begin{array}{l}\text { La Sociedad Internacional de Trombosis y Hemostasia y la } \\
\text { Sociedad Estadounidense de Hematología, recomiendan que } \\
\text { todos los pacientes hospitalizados con COVID19, incluidos los } \\
\text { pacientes no UCl, reciban dosis profilácticas de HBPM a menos } \\
\text { que estén contraindicadas }{ }^{51,52} \text {. } \\
\text { La UFH también puede considerarse para la tromboprofilaxis. }\end{array}$ \\
\hline
\end{tabular}

Tomado de: Pharmacists Advancing Healthcare. Assessment of Evidence for COVID-19-Related Treatments. 2020. Disponible en: https://www.ashp.org/-I media/assets/pharmacy-practice/resource-centers/Coronavirus/docs/ASHP-COVID-19-Evidence-Table.ashx. Se efectuaron modificaciones. https:// creativecommons.org/licenses/by/4.0/

\section{Conclusiones}

En todo el mundo, el pilar del tratamiento de COVID-19 es la terapia de soporte. A la fecha, no hay un medicamento específico que reduzca la mortalidad provocada por la neumonía, la respuesta inflamatoria excesiva y/o la hipercoagulabilidad, que produce el SARS-CoV-2.

Desde el inicio de la pandemia los profesionales de la salud han utilizado medicamentos como la cloroquina, hidroxicloroquina, azitromicina, remdesivir, lopinavir/ ritonavir, esteroides e inhibidores de interleucina-6, como uso compasivo de drogas.
Lo anterior, con base en la extrapolación de estudios in vitro que demostraron las propiedades antivirales y antiinflamatorias de estos fármacos. Propiedades que no han sido demostradas de manera robusta en el ámbito clínico, a través de ensayos controlados aleatorizados.

El enfoque de "¿qué tienes que perder?", una posición común de individuos o familias desesperadas, debe ser equilibrado con el dictamen del juramento hipocrático: primero, no hacer daño; tomando en cuenta los efectos adversos que podrían develarse al utilizar fármacos off label durante una pandemia, los cuales no fueron detectados previamente debido al pequeño número de pacientes expuestos. 
Solo la cloroquina, la hidroxicloroquina, el remdesivir y el favipiravir, han recibido autorización de uso de emergencia para ser utilizados en pacientes con COVID-19, y en el caso de los primeros dos, estos ya fueron suspendidos como profilácticos o terapéuticos contra COVID-19. Ningún otro medicamento ha recibido autorización alguna a la fecha, y ninguno, inclusive el remdesivir y el favipiravir, poseen aprobación definitiva como terapéuticos contra COVID-19.

\section{Referencias Bibliográficas}

1. Huang C, Wang Y, Li X, Ren L, Zhao J, Hu Y, et al. Clinical features of patients infected with 2019 novel coronavirus in Wuhan, China. The Lancet. 2020;395(10223):497-506. DOI: $10.1016 /$ S0140-6736(20)30183-5

2. Li H, Zhou Y, Zhang M, Wang H, Zhao Q, Liu J. Updated approaches against SARSCoV-2. Antimicrob Agents Chemother. 2020. DOI: 10.1128/AAC.00483-20

3. Cao W, Liu X, Bai T, Fan H, Hong K, Song H, et al. High-Dose Intravenous Immunoglobulin as a Therapeutic Option for Deteriorating Patients With Coronavirus Disease 2019. Open Forum Infectious Diseases. 2020;7(3):1-6. DOI: 10.1093/ofid/ofaa102

4. Siddiqi HK, Mehra MR. COVID-19 IIIness in Native and Immunosuppressed States: A Clinical-Therapeutic Staging Proposal. The Journal of Heart and Lung Transplantation. 2020. DOI: 10.1016/j.healun.2020.03.012

5. Russell CD, Millar JE, Baillie JK. Clinical evidence does not support corticosteroid treatment for 2019-nCoV lung injury. The Lancet. 2020;395(10223):473-5. DOI: 10.1016/S0140-6736(20)30317-2

6. Shang L, Zhao J, Hu Y, Du R, Cao B. On the use of corticosteroids for 2019- nCoV pneumonia. The Lancet. 2020;395(10225):683-4. DOI: 10.1016/S0140-6736(20)30361-5

7. Mehta P, McAuley DF, Brown M, Sanchez E, Tattersall RS, Manson JJ. COVID-19: consider cytokine storm syndromes and immunosuppression. The Lancet. 2020;395(10229):1033-4. DOI: $10.1016 /$ S0140-6736(20)30628-0

8. Jawhara S. Could Intravenous Immunoglobulin Collected from Recovered Coronavirus Patients Protect against COVID-19 and Strengthen the Immune System of New Patients? IJMS. 2020;21(7):2272. DOI: 10.3390/ijms21072272

9. Kruse RL. Therapeutic strategies in an outbreak scenario to treat the novel coronavirus originating in Wuhan, China. F1000Res. 2020;9:72. DOI: $10.12688 /$ f1000research.22211.2.
10. Roback JD, Guarner J. Convalescent Plasma to Treat COVID-19: Possibilities and Challenges. JAMA. 2020. DOI: $10.1001 /$ jama.2020.4940

11. Shen C, Wang Z, Zhao F, Yang Y, Li J, Yuan J.Treatment of 5 Critically III Patients with COVID-19 with Convalescent Plasma. JAMA. 2020. DOI: $10.1001 /$ jama.2020.4783

12. Hoffmann M, Kleine-Weber H, Schroeder S, Krüger N, Herrler T, Erichsen S, et al. SARS-CoV-2 Cell Entry Depends on ACE2 and TMPRSS2 and Is Blocked by a Clinically Proven Protease Inhibitor. Cell. 2020. DOI: $10.1016 /$ j.cell.2020.02.052

13. Vincent MJ, Bergeron E, Benjannet S. Chloroquine is a potent inhibitor of SARS coronavirus infection and spread. Virol J. 2005;2(69):1-10. DOI: 10.1186/1743-422X2-69

14. Yan Y, Zou Z, Sun Y, Li X, Xu K-F, Wei Y, et al. Anti-malaria drug chloroquine is highly effective in treating avian influenza A H5N virus infection in an animal model. Cell Res. 2013;23(2):300-2. DOI: 10.1038/cr.2012.165

15. U.S. Department of Health and Human Service, Food and Drug Administration. Coronavirus (COVID-19) Update:

Daily Roundup. 2020. Disponible en: https://www.fda.gov/news-events/ pressannouncements/coronavirus-covid19-update-daily-roundup-march-30-2020

16. U.S. Department of Health and Human Service, Food and Drug Administration. FDA cautions against use of Hydroxychloroquine or Chloroquine for COVID-19 outside of the hospital setting or a clinical trial due to risk of heart rhythm problems. 2020. Disponible en: https://www.fda.gov/drugs/drug-safetyand-availability/fdacautions-against-usehydroxychloroquineor-chloroquine-covid19-outside-hospitalsetting-or

17. Blaising J, Polyak SJ, Pécheur E-I. Arbidol as a broad-spectrum antiviral: An update. Antiviral Research. 2014;107:84-94. DOI: 10.1016/j.antiviral.2014.04.006

18. McCreary EK, Pogue JM. COVID-19 Treatment: A Review of Early and Emerging Options. Open Forum Infectious Diseases. 2020;7(4):1-11. DOI: 10.1093/ofid/ofaa105

19. Warren TK, Jordan R, Lo MK, Ray AS, Mackman RL, Soloveva V. et al. Therapeutic efficacy of the small molecule GS-5734 against Ebola virus in rhesus monkeys. Nature. 2016;531(7594):381-5. DOI: 10.1038/ nature 17180

20. Al-Tawfiq JA, Al-Homoud AH, Memish ZA. Remdesivir as a possible therapeutic option for the COVID-19. Travel Med Infect Dis. 2020;101615. DOI: 10.1016/j. tmaid.2020.101615

21. Wang M, Cao R, Zhang L, Yang X, Liu J, Xu M, et al. Remdesivir and chloroquine effectively inhibit the recently emerged novel 
coronavirus (2019-nCoV) in vitro. Cell Res. 2020; 30(3):269-71. DOI: 10.1038/s41422020-0282-0

22. Williamson BN, Feldmann F, Schwarz B, Meade-White K, Porter DP, Schulz $J$, et al. Clinical benefit of remdesivir in rhesus macaques infected with SARS-CoV-2. Microbiology. 2020. DOI: $10.1101 / 2020.04 .15 .043166$

23. Grein J, Ohmagari N, Shin D, Diaz G, Asperges E, Castagna A, et al. Compassionate Use of Remdesivir for Patients with Severe Covid-19. N Engl J Med. 2020. DOI: 10.1056/NEJMoa2007016

24. U.S. Department of Health and Human Service, Food and Drug Administration. Coronavirus (COVID-19): FDA Issues an Emergency Use Authorization for Potential COVID-19 Treatment. 2020. Disponible en: https://www.fda.gov/media/137564/ download

25. Furuta Y, Komeno T, Nakamura T. Favipiravir (T-705), a broad-spectrum inhibitor of viral RNA polymerase. Proceedings of the Japan Academy Ser B: Physical and Biological Sciences. 2017;93(7):449-63. DOI: 10.2183/ pjab.93.027

26. Dong L, Hu S, Gao J. Discovering drugs to treat coronavirus disease 2019 (COVID-19). Drug Discoveries \& Therapeutics. 2020;14(1):58-60. DOI: $10.5582 /$ ddt.2020.01012

27. Morse JS, Lalonde T, Xu S, Liu WR. Learning from the Past: Possible Urgent Prevention and Treatment Options for Severe Acute Respiratory Infections Caused by 2019nCoV. Chem Bio Chem. 2020;21 (5):730-8. DOI: $10.1002 /$ cbic.202000047

28. World Health Organization. (2020). WHO R\&D Blueprint: informal consultation on prioritization of candidate therapeutic agents for use in novel coronavirus 2019 infection, Geneva, Switzerland, 2020. World Health Organization. Disponible en: https:// apps.who.int/iris/handle/10665/330680

29. Caly L, Druce JD, Catton MG, Jans DA, Wagstaff KM. The FDA-approved Drug Ivermectin inhibits the replication of SARSCoV-2 in vitro. Antiviral Res. 2020 DOI: 10.1016/i.antiviral.2020.104787

30. Jiang S, Liu T, Hu Y, Li R, Di X, Jin X, et al. Efficacy and safety of glucocorticoids in the treatment of severe community-acquired pneumonia. Medicine. 2019;98(26):e16239. DOI: 10.1097/MD.0000000000016239

31. Zhou, W., Liu, Y., Tian, D. Potential benefits of precise corticosteroids therapy for severe 2019-nCoV pneumonia. Sig Transduct Target Ther. 2020;5:18. DOI: 10.1038/s41392-0200127-9

32. Zhang C, Wu Z, Li J-W, Zhao H, Wang G-Q. The cytokine release syndrome (CRS) of severe COVID-19 and Interleukin-6 receptor (IL-6R) antagonist Tocilizumab may be the key to reduce the mortality. Int J Antimicrob Agents. 2020. DOI: 10.1016/j. ijantimicag.2020.10595439

33. Baron SA, Devaux C, Colson P, Raoult D, Rolain JM. Teicoplanin: an alternative drug for the treatmentof coronavirus COVID-19? International Journal of Antimicrobial Agents. 2020. DOI: 10.1016/j. ijantimicag.2020.105944

34. Choy K-T, Wong AY-L, Kaewpreedee P, Sia SF, Chen D, Hui KPY, et al. Remdesivir, lopinavir, emetine, and homoharringtonine inhibit SARS-CoV-2 replication in vitro. Antiviral Research. 2020;178:1-5. DOI: 10.1016/j. antiviral.2020.104786

35. World Health Organization. The use of nonsteroidal anti-inflammatory drugs (NSAIDs) in patients with COVID-19. 2020. Disponible en: https://www.who.int/publicationsdetail/the-use-of-nonsteroidal-antiinflammatory-drugs-(nsaids)-in-patientswith-covid-19

36. National Health Service. How to treat coronavirus symptoms at home. 2020. Disponible en: $\underline{\text { https://www.nhs.uk/ }}$ conditions/coronavirus-covid-19/ whatto-do-if-you-or-someone-you-livewithhas-coronavirus-symptoms/how-totreatcoronavirus-symptoms-at-home/

37. European Medicines Agency. EMA gives advice on the use of non-steroidal antiinflammatories for COVID-19. 2020. Disponible en: https://www.ema.europa. eu/en/news/ema-gives-advice-usenonsteroidal-anti-inflammatories-covid-19

38. U.S. Food \& Drug Administration. FDA advises patients on use of non-steroidal anti-inflammatory drugs (NSAIDs) for COVID-19. March 19, 2020. Disponible en: https://www.fda.gov/drugs/drugsafetyand-availability/fda-advises-patientsusenon-steroidal-anti-inflammatorydrugsnsaids-covid-19

39. Chen T, Wu D, Chen H, Yan W, Yang D, Chen $\mathrm{G}$, et al. Clinical characteristics of 113 deceased patients with coronavirus disease 2019: retrospective study. BMJ. 2020; DOI: 10.1136/bmj.m1091

40. Wang D, Hu B, Hu C, Zhu F, Liu X, Zhang J, et al. Clinical Characteristics of 138 Hospitalized Patients With 2019 Novel Coronavirus- Infected Pneumonia in Wuhan, China. JAMA. 2020;323(11):10611069. DOI: $10.1001 /$ jama.2020.1585

41. Zhou F, Yu T, Du R, Fan G, Liu Y, Liu Z, et al. Clinical course and risk factors for mortality of adult inpatients with COVID-19 in Wuhan, China: a retrospective cohort study. Lancet. 2020;395(10229):1054-1062. DOI: $10.1016 /$ S0140-6736(20)30566-3

42. Klok FA, Kruip MJHA, van der Meer NJM, Arbous MS, Gommers DAMPJ, Kant KM, 
et al. Incidence of thrombotic complications in critically ill ICU patients with COVID-19.

Thromb Res. 2020. DOI: 10.1016/j.

thromres.2020.04.013

43. Tang N, Bai H, Chen X, Gong J, Li D, Sun Z. Anticoagulant treatment is associated with decreased mortality in severe coronavirus disease 2019 patients with coagulopathy. Journal of Thrombosis and Hemostasis. 2020;18(5):1094-9. DOI: 10.1111/jth.14817

44. World Health Organization. Guidance for managing ethical issues in infectious disease outbreaks. 2016. Disponible en: https://apps.who.int/iris/bitstream/han dle/10665/250580/9789241549837-eng. pdf? sequence $=1$ \&isAllowed $=y$

45. Zhou D. COVID-19: a recommendation to examine the effect of hydroxychloroquine in preventing infection and progression. J Antimicrob Chemother. 2020. DOI: 10.1093/ $\mathrm{jac} / \mathrm{dkaa} 114$

46. Yao $X$, Ye F, Zhang M. In vitro antiviral activity and projection of optimized dosing design of hydroxychloroquine for the treatment of Severe Acute Respiratory Syndrome Coronavirus 2 (SARS-CoV-2). Clin Infect Dis. 2020. DOI: $10.1093 / \mathrm{cid} / \mathrm{ciaa237}$

47. Liu J, Cao R, Xu M. Hydroxychloroquine, a less toxic derivative of chloroquine, is effective in inhibiting SARS-CoV-2 infection in vitro. Cell Discov. 2020;6:1-4. DOI: 10.1038/ s41421-020-0156-0

48. Ohe M. Macrolide treatment for COVID-19: will this be the way forward? Biosci Trends. 2020. DOI: $10.5582 /$ bst.2020.03058

49. Tran DH. Azithromycin, a 15-membered macrolide antibiotic, inhibits influenza A (H1N1) pdm09 virus infection by interfering with virus internalization process. J Antibiot. 2019;72:759-768. DOI: $\underline{10.1038 / 541429-019-}$ 0204-x

50. Retallack H. Zika virus cell tropism in the developing human brain and inhibition by azithromycin. Proc Natl Acad Sci USA. 2016;113:14408-14413. DOI: 10.1073/ pnas.1618029113

51. American Society of Hematology. COVID-19 and coagulopathy: frequently asked questions. 2020. Disponible en: $\underline{\text { https:// }}$ www.hematology.org/covid19/covid-19and-coagulopathy

52. International Society of Thrombosis and Hemostasis Interim Guidance on Recognition and Management of Coagulopathy in COVID-19. 2020. Disponible en: https://onlinelibrary.wiley. com/doi/epdf/10.1111/jth.14810

53. National Institutes of Health Coronavirus Disease 2019 (COVID-19) Treatment Guidelines NIH website. 2020. Disponible en: https://www.covid19treatmentguidelines. nih.gov/

54. Infectious Diseases Society of America (IDSA) guidelines on the treatment and management of patients with COVID-19. IDSA website. 2020. Disponible en: https:// www.idsociety.org/globalassets/idsa/ practice-guidelines/covid-19/treatment/ idsa-covid-19-gl-tx-and-mgmt-v1.0.4.pdf

55. U.S. Department of Health and Human Service, Food and Drug Administration, Center for Biologics Evaluation and Research. Investigational COVID-19 convalescent plasma guidance for industry. 2020. Disponible en: https://www.fda.gov/ media/136798/download 